\title{
Study on the Technical Characteristics and Development Direction of 4G Communication
}

\author{
Dehou Liang, Yang Zhang \\ Beijing Information Technology College, Beijing, 100070, China
}

\begin{abstract}
With the rapid development of electronic information technology, future mobile communication technology would be characterized by faster speed, wider spectrum, more flexible and intelligent operation, better compatibility, high quality and low cost. This paper mainly describes the main technical characteristics and development direction of 4G mobile communication technology.
\end{abstract}

Keywords-4G communication; technical characteristics; development direction.

\section{INTRODUCTION}

4G is short for 4rd Generation, and its Chinese meaning is the fourth generation of digital communication. The fourth generation of digital communication has asymmetric data transmission capacity greater than $2 \mathrm{Mbps}$, the data rate is over UMTS, is the ideal model to support high-speed data rate (2 20Mbps) connection, the connection speed can be increased from $2 \mathrm{Mbps}$ to $100 \mathrm{Mbps}$, which is equivalent to $50 \mathrm{x}$ the third generation of digital communication technology, and can transmit high-quality 3D images. Information transferring level of $4 \mathrm{G}$ communication technology is one level higher than that of 3G, and also has the ability to automatically switch between different rates. The anti-attenuation performance of $4 \mathrm{G}$ signal is better than that of 3G, and its frequency utilization efficiency is much higher than that of 3G. In terms of capacity, due to the introduction of spatial division multiple access (SDMA) technology, its capacity is up to 10 times larger than that of 3G. On the basis of $3 \mathrm{G}$ communications technology, 4G technology uses some new technologies to improve and perfect the efficiency and functionality of $3 \mathrm{G}$ wireless communication network. 4G technology is qualified with higher data rate, spectral efficiency, security, intelligence, flexibility, higher transmission quality and service quality.

\section{The MAIN TECHNICAL CHARACTERISTICS OF 4G COMMUNICATION}

\section{A. Faster communication speed, wider network spectrum}

The feature of $4 \mathrm{G}$ communication is that its communication speed is much faster than that of $3 \mathrm{G}$ communication. The first generation applies analog communications technology, and it only provides voice services; transmission rate of the second-generation digital communications is $9.6 \mathrm{Kbps} \sim 32 \mathrm{Kbps}$; although thirdgeneration digital communication's transmission rate can reach $2 \mathrm{Mbps}$, the transmission rate of $4 \mathrm{G}$ is expected to $100 \mathrm{Mbps} \sim 150 \mathrm{Mbps}$ or even higher. In order to get $4 \mathrm{G}$ communication achieve higher transmission rate, upgrading and rebuilding should be conducted on the basis of existing $3 \mathrm{G}$ communication network, so as to make the bandwidth of 4G communication network to reach its technical requirements. According to current research technology of $4 \mathrm{G}$, it is expected that each $4 \mathrm{G}$ channel will occupy $100 \mathrm{MHz}$ spectrum, which is equivalent to about 20 times of W-CDMA $3 G$ network.

\section{B. Higher frequency utilizing efficiency}

Compared to 3G, 4G applies many powerful breakthrough technology. For example, to further improve the bandwidth of the backbone network, 4G introduces exchange hierarchy technology, which can simultaneously cover different types of communication interfaces; furthermore, 4G also uses routing technology-based network architecture. Due to the use of several different technologies, the frequency utilizing efficiency of $4 \mathrm{G}$ is much higher than that of the third generation, $4 \mathrm{G}$ also has faster speed, and its download speed may reach $5 \mathrm{Mbps}$ to $10 \mathrm{Mbps}$.

\section{More flexible communication and higher intelligence}

4G can access the Internet at any place and any time, can provide communication service of $150 \mathrm{Mbps}$ speed for users, and can provide integrated functions of positioning, timing, data acquisition and remote control. Its application will be more extensive, such as video conferencing, wireless Internet and so on. Users can also connect a variety of electronic devices to $4 \mathrm{G}$ system, to realize the diversification of $4 \mathrm{G}$ network service, and form the bridge of communication for multiple sectors and multiple systems. $4 \mathrm{G}$ communication will enable us to communicate at any place and any time, and transmit data, voice and images in two directions to conduct on-line real-time communication. Future 4G mobile phone will have major breakthrough in both appearance and style. Let us wait and see what happens.

4G communication has higher intelligence. This is not only due to that the design and operation of $4 \mathrm{G}$ terminal equipment are intelligent, more importantly, $4 \mathrm{G}$ phones can realize many unimaginable functions, such as mobile TV, job alerts, live secretary, 'mobile electronic money', ecommerce and so on. 


\section{Better compatibility and higher quality multimedia communication}

4G communication can combine different communication modes with the terminal, such as television broadcast communication, wireless local area network communication, mobile communication, radio communication, and satellite communication.

For $4 \mathrm{G}$ communication to be accepted by the majority of mobile phone users as soon as possible, in addition to having powerful functions, the existing communication infrastructure should also be considered, making the majority of mobile phone users transit to $4 \mathrm{G}$ with less investment.

4G will not only provide a clear voice quality, but also provide high-resolution images and other multimedia transmission. Although the third generation of mobile communication system can achieve a variety of multimedia communications, 4G communication has a greater advantage on coverage area, communication quality and cost performance, and can meet the multimedia service requirements of high speed, high resolution and large amounts of data. Multimedia communication services supplied by future 4G will involve areas of news, entertainment, advertising, education, etc., to achieve the high-speed and high-fidelity transmission of data, voice, images and other information. Therefore, the future 4G communication system is also known as "multimedia mobile communication", which can improve the existing communication quality and hold a large number of users to conduct high-speed data transmission.

\section{E. Cheaper communication costs}

4G communication network operators will consider about upgrading on the basis of 3G communication network directly, to be compatible with $3 \mathrm{G}$ communication, so as to effectively reduce the cost of operators and users. 4G will adopt flexible pricing strategies to meet different customers' needs, for example, paying as daily, monthly subscription, packages, and subscribing for one year, two years or even longer time, to enjoy more benefits and services, making the cost of $4 \mathrm{G}$ communication services be cheaper than that of $3 \mathrm{G}$ communication.

\section{APPLICATION OF OFDM TECHNOLOGY IN THE 4G}

4G communication doesn't evolve from simple upgrading on the basis of original $3 \mathrm{G}$ communication technology, besides, their core technologies are very different. Main core technology of 3G communication system is CDMA, while $4 \mathrm{G}$ communication system applies orthogonal frequency division multiplexing (OFDM) technology which is a single-band receiver with the advantages of reducing or eliminating the interference between the signals, improving the spectrum utilization and realizing low cost. Using the above techniques, 4G can achieve the value-added services of digital audio broadcasting (DAB) and wireless local area loop (WLL). Taking into account the transition and compatibility problems of $3 \mathrm{G}$ communication and 4G communication, 4G communication system not only applies OFDM technology, but also needs to combine both CDMA technology and OFDM technology together to conduct technology integration, such as OFDM / CDMA technology, in order to make $4 \mathrm{G}$ be better compatible with 3G. Digital audio broadcasting (DAB) system aforementioned applies a combination of both OFDM and FDMA technologies, that is, OFDM / FDMA technology. It is expected that future 4G communication system will apply OFDM technology as the core, and integrate and apply the advantages of OFDM and CDMA technologies.

\section{DEVELOPMENT DiRECTION}

Currently, the number of global $4 \mathrm{G}$ mobile phone users has reached nearly 50 million, and the number of global 4G mobile phone users is expected to more than 1 billion in 2016. In China, the technical standard, terminal equipment, network equipment and frequency allocation of 4G have been basically ready. So far, the domestic TD-LTE (4G technology) network has covered more than 100 cities, and large-scale 4G experience is started in Shenzhen and Guangzhou. Due to the popularity of 3G technology, more and more people access the Internet with mobile phones, according to statistics, the world's mobile phone users have reached as much as 4.5 billion until 2013, the number of mobile Internet users reaches nearly 1.8 billion, and the number of smart mobile phones is also growing by leaps and bounds.

Mobile communication implements the interconnection between people, and also is realizing the interconnection between people and the Internet. In the future, mankind will usher the era of Internet of Things of interconnection between people and things and between things. It is expected that after 10 years the world will have billions of items be interconnected. By then, the usages of mobile phone will be greatly increased, and broadband, individuation, intelligence, multi-function, media, environmental protection are new trends of mobile communication development.

4G communication not only provides the freedom for people to communicate, and will profoundly change the way people live, which has broad application areas and markets. In the future, it will be widely used in smart family, mobile health care, smart classroom, live TV, education and scientific research, intelligent agriculture and smart phones .

\section{CONCLUSION}

4G means the integration of more technologies, industries and application, and 4G will no longer be confined to the telecommunications industry, is also used in finance, health care, education, transportation and other industries. 4G technology will evolve into wireless broadband, its communication terminal handset will not only be a communication tool, but also can do more things, for example, multimedia communication besides voice communication, remote control; a broadcast network integrated by LAN, Internet, telecommunications networks, radio networks, satellite networks and other components. In 
short, regardless of whatever the terminal applied is, we can enjoy high-quality information services, to enable $4 G$ to penetrate into every aspect of our lives.

\section{REFERENCES}

[1] Yanbin Liu. Beacon Leading the Development of China's Railway Communication - The Fourth Generation of Wireless Communication Technology (4G). Technology Wind, 2010.
[2] Kai Hu. Reflections on Smart Phone System Under 4G Standard. Science and Technology and Enterprise, 2012.

[3] Tao Yang. Key Technologies and Prospects Discussion of 4G Network Development. Information and Communication, 2013.

[4] Lan Cheng. Key Technologies Characteristics and Prospects Analysis of 4G Network Development. Network Security Technology \& Application , 2014. 